\title{
BMJ Open Couples-based approach to HIV prevention for transgender women and their partners: study protocol for a randomised controlled trial testing the efficacy of the 'It Takes Two' intervention
}

\author{
Kristi E Gamarel, ${ }^{1}$ Jae M Sevelius, ${ }^{2,3}$ Torsten B Neilands, ${ }^{2,3}$ Rachel L Kaplan, ${ }^{4}$
} Mallory O Johnson, ${ }^{2,3}$ Tooru Nemoto, ${ }^{5}$ Lynae A Darbes, ${ }^{6}$ Don Operario (D) ${ }^{7}$

To cite: Gamarel KE, Sevelius JM, Neilands TB, et al. Couples-based approach to HIV prevention for transgender women and their partners: study protocol for a randomised controlled trial testing the efficacy of the 'It Takes Two' intervention. BMJ Open 2020;10:e038723. doi:10.1136/ bmjopen-2020-038723

- Prepublication history and additional material for this paper is available online. To view these files, please visit the journal online (http://dx.doi.org/10. 1136/bmjopen-2020-038723)

Received 20 March 2020 Revised 27 August 2020 Accepted 09 September 2020

Check for updates

(c) Author(s) (or their employer(s)) 2020. Re-use permitted under CC BY-NC. No commercial re-use. See rights and permissions. Published by BMJ.

For numbered affiliations see end of article.

Correspondence to Dr Don Operario; Don_0perario@brown.edu

\section{ABSTRACT}

Introduction HIV transmission and acquisition risk among transgender women is particularly high in the context of primary partnerships. This project extends a previous pilot couples-focused HIV intervention programme, which was shown to be feasible, acceptable and promising in reducing sexual risk behaviour among transgender women and their partners. This randomised controlled trial (RCT) tests the efficacy of this culturally sensitive HIV prevention programme for HIV-serodiscordant and HIV-negative seroconcordant transgender women and their partners. Methods and analysis To finalise the protocol for trial, we used qualitative methods to hone eligibility criteria, refine the intervention and control manuals, and name and brand the intervention ('It Takes Two'). The RCT investigates the effects of the It Takes Two intervention on Composite Risk for HIV (CR-HIV) among 100 couples. CR-HIV is a binary indicator of couple HIV risk using validated measures of sexual behaviour, pre-exposure prophylaxis use among HIV-negative participants and viral suppression among participants living with HIV. Using a two-arm RCT, we will examine intervention effects on CRHIV at 12-month follow-up comparing transgender women and their partners randomised to the intervention versus control (HIV prevention information only).

Ethics and dissemination This study has been reviewed and approved by the University of California, San Francisco (19-28624) and the University of Michigan (HUM00147690) Institutional Review Boards. Participants provide informed consent before taking part of the study activities. Results will be published in peer-reviewed journals and presented at scientific conferences. We will make our results available to the community of researchers and general public interested in transgender health to avoid unintentional duplication of research, as well as to others in the health and social services community, including LGBT community-based organisations, AIDS service organisations and other transgender-serving organisations. The full de-identified dataset and codebook will be shared at the University of Michigan Digital Repository.
Strengths and limitations of this study

- Our primary outcome measure is Composite Risk for HIV, which uses multiple validated indicators of HIV risk and acknowledges the diversity of HIV prevention needs that couples might have and desire.

- We include both seroconcordant-negative and serodiscordant couples in order to test the intervention with both types of dyads in which HIV transmission risk might occur.

- Study design is not powered to detect effects on HIV incidence; seroconcordant-positive couples are excluded from this study; contamination between intervention arms may occur due to close social networks in the transgender community.

Trial registration number NCT04067661.

\section{INTRODUCTION}

Background and rationale

Transgender women (individuals with a feminine and/or female gender identity who were assigned men at birth and hereafter 'trans women') are disproportionately affected by HIV. Trans women represent one of the populations at highest risk for HIV infection, both in the USA and globally. ${ }^{1}$ Two independent meta-analyses of HIV prevalence studies in trans women estimated that $19 \%$ of trans women are living with $\mathrm{HIV}^{23}$ and reports of HIV incidence ${ }^{24}$ and prevalence rates ${ }^{5}$ are among the highest of all risk groups. ${ }^{2}$ One of the most consistently reported contexts for HIV transmission among trans women is within a primary partnership with a cisgender man (hereafter 'cis man'). ${ }^{6-10}$ HIV prevention interventions addressing relationship 
contexts for trans women and their primary partners are needed. $^{11}$

Couples-based HIV prevention interventions have been shown to be feasible, acceptable and efficacious in reducing sexual risk among heterosexual couples and men who have sex with men. ${ }^{12-15}$ No efficacious couplesbased HIV interventions for trans women and their partners currently exist. Previous research has identified intervention targets for reducing HIV transmission in trans women and cis men couples. ${ }^{7-9}{ }^{16-21}$ Identified risk factors among these dyads include condomless sex within the partnership and with outside partners, mental health, substance use and stigma toward the relationship. ${ }^{19} 22$ Communication barriers about condom use with outside partners were also identified. ${ }^{23-25}$

We previously developed a couples-based HIV prevention programme for trans women and cis men in a primary partnership (called 'Couples HIV Intervention Programme' or CHIP). A pilot study found that CHIP was feasible, acceptable and produced significant reductions in condomless sex acts with primary and casual partners and in number of casual partners at 3-month follow-up compared with a control group. ${ }^{20}$ The protective effects of pre-exposure prophylaxis (PrEP) and an undetectable viral load on HIV transmission had not yet been established when CHIP was piloted, so the intervention lacked content on these biomedical HIV prevention strategies for trans women and their partners. ${ }^{26-28}$

The current trial will test the efficacy of a couples-focused HIV prevention programme for HIV serodiscordant and HIV-negative seroconcordant couples of trans women and their primary partners, renamed by trans community members as It Takes Two. The primary outcome is a novel Composite Risk for HIV (CR-HIV) measure, which is a binary indicator of couple HIV risk incorporating validated measures of (1) sexual behaviour (defined as condomless anal or vaginal sex with a serodiscordant or unknown HIV status primary or outside partner), (2) preexposure prophylaxis (PrEP) use among HIV-negative participants and (3) viral suppression among participants living with HIV. ${ }^{29}$ Using a two-arm randomised controlled trial (RCT), this study will examine intervention effects on CR-HIV at quarterly time points over a year comparing 100 couples of trans women and their primary partners randomised to the It Takes Two intervention versus HIV prevention information only (50 couples per group).

\section{Objectives}

The primary specific aim is to:

Evaluate the efficacy of It Takes Two on CR-HIV compared with an HIV prevention information control condition.

We hypothesise that couples in the intervention condition will have lower CR-HIV at 12-month follow-up compared with couples in the control condition.

The secondary aims are to:

Assess the effects of the intervention on theory-based mediators (ie, communication and collaborative coping skills); and explore partner gender identity and couple HIV status as moderators of the effect of the intervention on CR-HIV. We hypothesise that couples in the intervention will report higher scores on the theory-based mediators, which will in turn be associated with lower CR-HIV at the 12-month follow-up compared with the couples in the control condition. The moderators analyses are exploratory; thus, there is no hypothesis of the effects of the intervention on CR-HIV.

\section{Trial design}

We conducted focus groups $(\mathrm{N}=2)$ with partnered trans women and in-depth interviews $(\mathrm{N}=4)$ with cis male partners to update and standardise intervention content to ensure that information and communication modules regarding biomedical prevention strategies were relevant and appropriate, and to develop training materials for peer health educators. Formative data indicated that trans women are increasingly engaging in HIV risk behaviours with partners who do not identify as cis men. Therefore, we expanded the eligibility criteria of trans women's partners to include all genders as long as there is HIV transmission or acquisition risk, either within the dyad or with outside partners. Qualitative findings also provided details on study protocol logistics (ie, input on the name of the study, flexible appointment hours specifically in evening hours, providing transportation compensation, conducting the study in discrete location).

We will recruit and randomise a total of 100 dyads (200 participants total) to test effects of the theory-based couples-focused intervention on CR-HIV. Couples in the control arm receive a single-session involving HIV prevention information, in which both partners separately view HIV prevention education videos and then receive referrals by a trained peer health educator. Couples in the intervention arm receive the 4-session It Takes Two intervention delivered by a peer health educator. All dyads are assessed quarterly over 12 months, and biological (ie, dried blood spots (DBSs) to test for PrEP adherence and viral load tests) and behavioural data are collected and analysed using state-of-the-science dyadic analyses.

\section{METHODS}

This protocol was prepared using the Standard Protocol Items: Recommendations for Interventional Trials reporting guidelines. ${ }^{30}$ The protocol was registered at ClinicalTrials.gov, and key protocol information is provided in table 1 in accordance with the WHO Trials Registration Data Set.

\section{Participants, interventions and outcomes \\ Study setting}

The study is conducted in San Francisco, California. ${ }^{31}$ Trans women have the overall highest HIV diagnosis rate of any group in California, ${ }^{32}$ with over half of new cases identified in San Francisco. ${ }^{33}$ Study activities take place at a community-based project site, the UCSF Community 
Table 1 Items from the WHO Trial Registration Data Set

\begin{tabular}{ll}
\hline Data category & Information \\
\hline $\begin{array}{l}\text { Primary registry and trial } \\
\text { identifying number }\end{array}$ & $\begin{array}{l}\text { ClinicalTrials.gov } \\
\text { NCT04067661 }\end{array}$ \\
$\begin{array}{l}\text { Date of registration in } \\
\text { primary registry }\end{array}$ & 26 August 2019 \\
\end{tabular}

Secondary identifying $\quad$ R01 MH115765
numbers

Sources of monetary or National Institute of Mental Health material support

\begin{tabular}{|c|c|c|}
\hline Primary sponsor & National Institute of Mental Health & files, including agreement to the \\
\hline
\end{tabular}
edu; (734) 647-3178)

Contract for scientific queries Kristi Gamarel PhD (kgamarel@umich. edu; (734) 647-3178)

$\begin{array}{ll}\text { Public title } & \text { 'It Takes Two' } \\ \text { Scientific title } & \begin{array}{l}\text { A couples-based approach to HIV } \\ \text { prevention for transgender women and } \\ \text { their male partner }\end{array}\end{array}$

Countries of recruitment USA

Health condition or HIV transmission risk problem studied

$\begin{array}{ll}\text { Intervention } & \text { Behavioural intervention: couples- } \\ \text { focused HIV prevention counselling }\end{array}$

Key inclusion and exclusion Age 18 or older

criteria Participants in a committed relationship and enrolling with their partner

One partner must identify as a trans woman

Both partners must have engaged in condomless sex within the past 6 months with any partner

At least one partner is HIV-negative, speak and read English

Exclusion criteria: currently psychotic, suicidal or manic; either partner reports that participation could cause them physical harm

\begin{tabular}{ll}
\hline Study type & $\begin{array}{l}\text { Interventional } \\
\text { Allocation: randomised } \\
\text { Intervention mode: parallel assignment } \\
\text { Masking: none } \\
\text { Primary purpose: prevention } \\
\text { Phase III }\end{array}$ \\
\hline Date of first enrolment & 1 November 2019 \\
\hline Target sample size & 200 \\
\hline Recruitment status & Recruiting \\
\hline Primary outcome(s) & $\begin{array}{l}\text { Composite indicator of HIV risk, } \\
\text { assessed at 3, 6, 9 and 12-month } \\
\text { follow-up }\end{array}$ \\
\hline Key secondary outcomes & $\begin{array}{l}\text { Communication, coping, goal } \\
\text { congruence }\end{array}$ \\
\hline Ethics review & $\begin{array}{l}\text { Approved 22 June 2018, Institutional } \\
\text { Review Boards at the University of } \\
\text { California, San Francisco (19-28624) } \\
\text { and approved by University of } \\
\text { Michigan (HUM00147690) }\end{array}$ \\
\hline N/A-study in progress
\end{tabular}

Continued
Table 1 Continued

\begin{tabular}{ll}
\hline Data category & Information \\
\hline Summary results & N/A-study in progress \\
IDP sharing statement & $\begin{array}{l}\text { After completion of the trial and } \\
\text { publication of primary research reports, } \\
\text { a deidentified individual clinical trial } \\
\text { participant-level analytic dataset } \\
\text { and codebook will be shared via a } \\
\text { download link located at the University } \\
\text { of Michigan Digital Repository. User } \\
\text { registration will be required to access } \\
\text { files, including agreement to the } \\
\text { conditions of use governing access to } \\
\text { the public release data }\end{array}$ \\
\hline
\end{tabular}

Research Center (UCRC) in the Tenderloin neighbourhood of San Francisco. The research team has administrative offices and access to private rooms to conduct interviews and intervention sessions, and the site is accessible via public transportation.

\section{Eligibility criteria}

For inclusion in the RCT, both individuals in the relationship must: (1) be age 18 or older; (2) be in a self-reported primary partnership with each other for at least 3 months (assessed through multiple questions validated in prior research); (3) have had penetrative anal or vaginal sex with the primary partner in the last 6 months; (4) be able to provide informed consent; and (5) be Englishspeaking. At least one individual in each couple must: (1) have had condomless sex with any partner in the last 6 months; and (2) be a trans woman. Couples must be HIV-negative concordant or HIV serodiscordant, in order to focus exclusively on HIV transmission risk. HIV status is verified at intake through standard individual HIV testing and counselling conducted at the study site; individuals who report being HIV-positive are asked to provide verification of their status. If one partner has a preliminary positive test result at their visit then they are referred to confirmatory testing. Staff members are trained to not reveal participants' HIV status results to their partners. The newly diagnosed participant has the option to disclose a positive test result to their partner. If they choose not to disclose, staff members will inform both partners that they are not eligible at this time but can screen for the study at a later date. We define a primary relationship as "currently in a relationship with someone you feel emotionally and sexually involved with for at least 3 months'. A 3-month time frame is based on our prior relationship research, indicating that many couples at risk for HIV are in the beginning of their relationship. ${ }^{22}$ Individuals are excluded if they: (1) are currently displaying symptoms of psychosis, or otherwise unable to complete informed consent procedures; (2) report that participation would be unsafe; or (3) have plans to move away from the area in the next 6 months. Excluded individuals are provided referrals to local resources (eg, mental health and couples counselling). To determine 
couple status, each member of the dyad is asked to answer a series of questions (eg, what pets, if any, do you have?, what pets, if any, does your partner have?) and answers are compared with verify couple status. ${ }^{3435}$

\section{Interventions}

The CHIP intervention protocol was adapted from Project Connect, a Centers for Disease Control and Prevention (CDC)-endorsed evidence based intervention developed with heterosexual couples. ${ }^{20}$ CHIP involved three counselling sessions lasting 60-90 min, and focused on providing information on HIV risk reduction strategies, communication and joint problem-solving in the relationship, supporting each other's health and the development of an HIV prevention plan.

The original CHIP protocol was updated based on qualitative findings to reflect current HIV prevention best-practice evidence by incorporating biomedical HIV prevention strategies, ${ }^{26-28}$ and content was refined to enhance relevance with racially/ethnically diverse trans women and their partners of different age groups. The final intervention, It Takes Two, was expanded to four sessions due to the inclusion of content about biomedical prevention, as well as additional skills training on communication related to HIV prevention.

Peer health educators deliver all of the intervention sessions. A licensed clinical social worker trained the peer health educators in delivering and maintaining fidelity to the It Takes Two manual over a 3-day training session. The training included specific strategies for working with couples (eg, managing conflict, attending to socialstructural barriers such as unemployment, financial strain and stigma). Peer health educators demonstrated proficiency via audiotaped role-plays prior to being 'certified' to deliver sessions. All counselling sessions are audiotaped (with participant informed consent), and peer health educators receive weekly supervision with the licensed clinical social worker, and the peer health educator complete a checklist after every session to indicate which activities were completed.

Table 2 provides an overview of the topics covered in the 4-session intervention programme. The first session is $20-30 \mathrm{~min}$ in length with the second, third and fourth sessions being approximately $60 \mathrm{~min}$ in length. Session are scheduled 1 week apart with a maximum of 2 weeks allowed between sessions.

Given that there is no standard of care for HIV prevention with trans woman and their partners, the control condition consists of participants viewing brief HIV prevention education videos on the following four topic areas: HIV testing; PrEP and post-exposure prophylaxis; condom use; and ' $\mathrm{U}=\mathrm{U}$ ' (undetectable=untransmittable). Participants are also provided with relevant referrals to HIV and other sexually transmitted infection (STI) prevention and care, mental health and social services. The videos last approximately $20 \mathrm{~min}$ and participants watch them immediately after their enrolment visit.

\section{Outcomes}

The primary outcome CR-HIV is a couple-level binary indicator of any HIV risk (eg, yes $=1$ vs no $=0$ ), which is derived from an algorithm based on whether one or both partners report condomless anal or vaginal sex with a HIV serodiscordant or status-unknown primary or other partner in the past 30 days. ${ }^{29}$ CR-HIV is assessed at baseline and every follow-up assessment. Couples are coded as 0 if neither partner reports condomless anal or vaginal sex. If one or both partners in the dyad report condomless

Table 2 It Takes Two counselling sessions

\begin{tabular}{|c|c|}
\hline Session and theme & Content \\
\hline Welcome and introduction & $\begin{array}{l}\text { Develop ground rules and boundaries } \\
\text { Identify relationship strengths } \\
\text { Set one relationship goal } \\
\text { - Amplify a positive moment in the relationship }\end{array}$ \\
\hline Couples communication & $\begin{array}{l}\text { Discuss communication styles } \\
\text { - Address differences in communication styles } \\
\text { - Practice new communication techniques } \\
\text { - Develop a plan for taking 'time outs' } \\
\text { - Set one relationship goal } \\
\text { - Amplify a positive moment in the relationship }\end{array}$ \\
\hline Couples HIV prevention & $\begin{array}{l}\text { Review sexual health and HIV prevention approaches, including biomedical HIV prevention } \\
\text { Identify current HIV prevention strategies } \\
\text { Learn about how to develop sexual agreements (eg, acceptability of having outside } \\
\text { partners) } \\
\text { Set one relationship goal } \\
\text { Amplify a positive moment in the relationship }\end{array}$ \\
\hline
\end{tabular}


sex with a serodiscordant or status-unknown primary or other partner, then the couple is coded as 1 if (1) the HIV-negative participant is not adherent to PrEP and/or (2) the partner living with HIV is not virally suppressed. Sexual risk behaviour is measured by the number of occasions of different sexual acts (vaginal, anal; receptive, insertive) with three different types of partners (primary, casual, paying partner), use of condoms during the past 30 days, and knowledge of partners' HIV status. At-risk sex is defined as any vaginal or anal intercourse without condoms that occurs with a person of known HIV-positive or unknown serostatus during the follow-up period. We assess PrEP use using DBS procedures for biological measurement of adherence from tenofovir diphosphate concentrations in blood plasma. ${ }^{36}$ At the start of the trial, we used dosing categories for quantification similar to those used in past drug-level studies of adherence to Truvada, ${ }^{37}$ allowing estimation of the number of tablets participants take per week (eg, 700-1249 fmol for 4-6xper week and $>1250$ for daily dosing). Participants also selfreport whether they are using 2-1-1 PrEP dosing and we have obtained an algorithm from our testing lab for assessing 2-1-1 strategy. The protocol was developed based on Truvada for PrEP, although we will note if participants are taking Descovy. Lab protocols including monitoring whether and when participants switch their regimen, which allows proper protocols for analysis, including noting washout periods. Participants living with HIV have blood drawn at each follow-up visit to determine viral suppression. Viral suppression is indicated by an undetectable HIV-1 level on the COBAS AmpliPrep/COBAS TaqMan HIV test kit (Roche Molecular Systems), which has a threshold for undetectability $\leq 20$ copies $/ \mathrm{mL}$ and is conducted at each follow-up assessment visit. A couple in which both partners are HIV-negative and only engage in condomless sex with one another would be coded as 0 regardless of whether either partner is on PrEP. Given budgetary constraints, we are unable to perform HIV and STI testing at the follow-up visits; however, our exploratory secondary outcomes include self-reported HIV and STI incidence over the 12 months of follow-up.

Participants complete the Conflicts Resolution Questionnaire, ${ }^{38}$ which includes mutual problem-solving skills. ${ }^{39}$ Collaborative communication is assessed using the Dyadic Coping Inventory ${ }^{40}$ which measures the extent to which couples work together, engage in mutual decision making and engage in open communication to alleviate a stressor (eg, HIV). Participants also complete a measure of sexual health goal congruence, which assesses the extent to which partners perceive they are the same page about their sexual health and HIV prevention plan. ${ }^{41}$

\section{Participant timeline}

The study started enrolment in November 2018 with a planned completion date of April 2023. Project activities occur over 12 months. Following baseline assessment, participants return for follow-up assessments at 3, 6, 9 and 12 months postrandomisation. Participants return for follow-up assessments regardless of relationship dissolution. Figure 1 provides a schematic diagram.

\section{Sample size}

Power analyses were generated using the two-group repeated proportions module in NCSS PASS V. $15^{42}$ based on formulas ${ }^{43}$ to compute a range of potential minimum detectable effect sizes for the primary analysis proposed to address hypothesis 1 . The CHIP pilot study found standardised effect sizes ranging from 0.38 to $0.42,{ }^{20}$ so we powered this study to detect similarly sized effects in worst-case scenarios where within-participant clustering of outcomes are substantial. The study will begin with 200 participants from 100 couples evenly assigned to the intervention and control groups. The CR-HIV outcome used to test hypothesis 1 will be measured at the individual level because individuals within dyadic partnerships can have different CR-HIV values based on their sexual behaviours with outside partners. Thus, the unit of analysis for testing hypothesis 1 will be repeated observations collected over time from individuals within dyads. Assuming 20\% attrition, data from 160 participants from 80 couples will be available for analysis at all time points. Attrition rates have ranged from $10 \%$ to $15 \%$ in our previous work with couples of trans women and men, with attrition typically due to male partners becoming incarcerated or relocation. To conservatively allow for the additional demands of intervention participation over 12 months, we estimated attrition to be $20 \%$ in this study. Due to the clustered nature of the dyadic data, observations from participants who belong to the same couple will be correlated. In a previous study of 190 trans women and their male partners, for instance, the average withincouple correlation of sexual risk measurements was $\mathrm{r}=0.07$. Accordingly, we lowered the effective sample size (ESS) input for the power analyses to be ESS=N/DEFF, where DEFF is the design effect or variance inflation attributable to using correlated data. DEFF is computed as $1+(\mathrm{M}-1) \times \mathrm{r}$, where $\mathrm{M}$ is the number of participants per dyad (ie, two). Therefore $\mathrm{DEFF}=1+(2-1) \times 0.07=1.07$, so $\mathrm{ESS}=160 / 1.07=149$. Assuming, $\alpha=0.05$, power $=0.80$ and $\mathrm{ESS}=149$, we computed the minimum detectable OR, proportion difference $\left(\mathrm{p}_{\text {diff }}\right)$ and standardised proportion difference $(h)$ for the time-averaged comparisons for the planned primary analyses, assuming four post-baseline measurements and a wide range of CR-HIV base rates $\mathrm{P}_{0}$ for levels of risk for trans women and their partners seen in the literature. ${ }^{784}$ Since the within-subject correlation $\rho$ for repeated measures is unknown, we varied it across a wide range from 0.20 to 0.80 . Minimum detectable ORs ranged from 0.17 to 0.56 ( 1.79 to 5.88 inverted) and minimum detectable raw proportions ranged from $6.6 \%$ to $20.5 \%$ resulting in corresponding minimum detectable standardised differences ranging from 0.27 to 0.42 . The obtained minimum detectable effect size estimates are similar to or smaller than those detected in our pilot study; this analytic sample provides statistical power to 


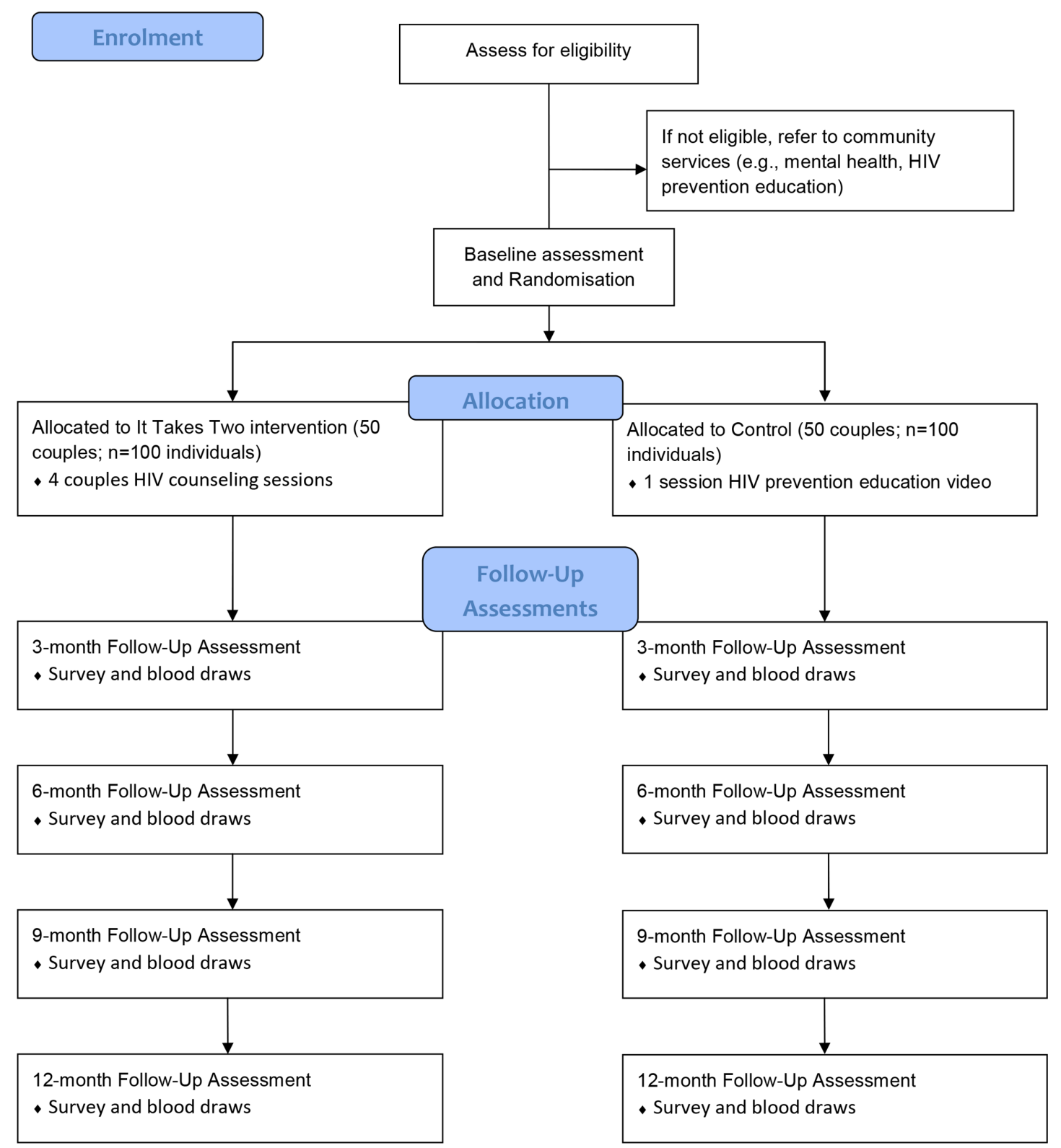

Figure 1 It Takes Two: schedule of enrolment, intervention and assessments.

detect the hypothesised effect on our primary outcome, CR-HIV.

\section{Recruitment}

We use a multifaceted approach to recruitment through online advertisements in social media, distributing flyers in targeted venues and outreach to agencies, clinics, bars, meal and shelter programmes, and single-room occupancy hotels.

Prospective participants call a toll-free recruitment line to for initial screening, and those who preliminarily qualify have their partner call the recruitment line. Once both partners preliminarily qualify, they both attend an in-person eligibility intake visit. While both partners must attend the initial appointment together, they are separated during their intake visit. We obtain informed consent from each partner and conduct a brief eligibility interview, which includes assessment of full inclusion criteria, as well as an HIV test (for those who do not provide documentation of HIV-positive status). Once both partners are deemed eligible and provide informed consent, they are scheduled for an enrolment visit to complete the remaining activities, including lab work, baseline assessment and randomisation.

Targeted recruitment goals were deemed feasible given the investigators' history of successful research with these populations (trans women and their partners), staff members' familiarity with community venues and support from community organisations in providing study referrals to their clients. The team will closely review enrolment targets and, in the case of recruitment difficulties and setbacks (eg, due to COVID-19 coinciding with the project period), will consider alternative strategies for participant recruitment and engagement. 


\section{Assignment of conditions}

\section{Allocation}

After completing the baseline survey, couples are randomised (stratified by couple serostatus at enrolment with equal numbers of seroconcordant vs serodiscordant couples) to the It Takes Two intervention or the HIV prevention information only control condition using a computerised secure and fraud-resistant procedure employed in prior studies. Researchers at University of California, San Francisco, have used this method in several RCTs; this method is successful in achieving balance across demographic, mediating and outcome variables. The project director independently generates the allocation sequence and assigns participants to trial arms; the research assistants enrol participants into the study. Neither the participants nor the research assistants will know of the allocation sequence in advance.

\section{Blinding}

Assessors are not blinded in this trial because they inform participants of their study condition.
Data collection, management, analysis

Data collection methods

Each potential participant completes a brief screening for gender identity (ie, trans woman, cis man), sex assigned at birth, age, race/ethnicity, sexual behaviour and relationship length. ${ }^{45-49}$ Each member of the couple is also asked whether participation would cause concern about physical or sexual harm. These data are used to determine eligibility. Table 3 summarises key measures.

Assessments are conducted in-person for each partner at baseline at 3, 6, 9 and 12-month follow-ups. Couples are scheduled to come in for assessments together, but assessment procedures are conducted separately to preserve the privacy of each individual. Under unique circumstances (eg, social isolation measures due to coronavirus), follow-up assessments are conducted remotely through online survey administration; in these instances, lab procedures are omitted from follow-up. Participants have both a couple ID and a participant ID so that individual data records can be dyadically linked. Participants complete all assessment regardless of whether they broke

\section{Table 3 It Takes Two outcomes and mediator variables}

\begin{tabular}{|c|c|}
\hline Construct & General description \\
\hline \multicolumn{2}{|l|}{ CR-HIV outcome } \\
\hline Sexual behavior ${ }^{64}$ & $\begin{array}{l}\text { Self-report condomless anal sex and condomless vaginal sex during the past } 30 \text { days, by type of partner: } \\
\text { main partner (yes/no), HIV-negative outside partners (yes/no), HIV-positive outside partners (yes/no), and } \\
\text { status-unknown outside partners (yes/no). }\end{array}$ \\
\hline $\begin{array}{l}\text { Antiretroviral Therapy (ART) } \\
\text { adherence and viral load }\end{array}$ & $\begin{array}{l}\text { Self-report ever missed taking HIV medications during the past } 30 \text { days (yes/no), how many days missed } \\
\text { at least one dose during the } 30 \text { days (\# days), 'how good a job did you do at taking your HIV medicine the } \\
\text { way you were supposed to' during the past } 30 \text { days ( } 0=\text { very poor to } 5=e x c e l l e n t) \text {, 'how often did you take } \\
\text { your HIV medicine the way you were supposed to' during the past } 30 \text { days ( } 0=\text { never to } 5=\text { always). Viral } \\
\text { load confirmation assessed via blood draws. }\end{array}$ \\
\hline PrEP use $\mathrm{e}^{2966}$ & $\begin{array}{l}\text { Self-report history of any lifetime PrEP use (yes/no), reason for taking PrEP (if relevant), PrEP use during } \\
\text { the past month (yes/no), current PrEP use (yes/no), reasons for PrEP discontinuation (if relevant), number } \\
\text { of PrEP pills taken every week during the past month (if relevant), frequency of missing PrEP pills during } \\
\text { the past month (if relevant; } 0=\text { never to } 3=\text { often), strategic PrEP dosing (yes/no), type of strategic dosing } \\
\text { schedule (if relevant), date of last dose of PrEP. PrEP adherence confirmation assessed via dried blood } \\
\text { spot. }\end{array}$ \\
\hline
\end{tabular}

\begin{tabular}{|c|c|}
\hline \multicolumn{2}{|l|}{ Secondary outcomes } \\
\hline $\begin{array}{l}\text { HIV and other Sexually } \\
\text { Transmitted Infection (STI) } \\
\text { history }^{67}\end{array}$ & $\begin{array}{l}\text { Self-report HIV status, with biologic assessment at baseline (for self-report baseline HIV-negative) or } \\
\text { medical confirmation (for self-report baseline HIV-positive). Self-report test for, test result, and treatment (if } \\
\text { relevant) during the past } 3 \text { months for: chlamydia, genital warts, gonorrhea, hepatitis B, hepatitis C, herpes } \\
\text { syphilis, trichomoniasis, other STI. }\end{array}$ \\
\hline \multicolumn{2}{|l|}{ Mediators } \\
\hline Goal congruence 6869 & $\begin{array}{l}\text { Self-report 4-item assessment regarding whether participants' perceive their sexual health goals are } \\
\text { aligned with their partners' sexual health goals (eg, 'How much do you feel like your main partner and you } \\
\text { are 'on the same page' in terms of the decisions you make about sexual health' rated on scale } 1=\text { not at all } \\
\text { to } 5=\text { a great deal). Self-report } 5 \text {-item assessment regarding whether participants and their partner have a } \\
\text { shared HIV prevention strategy (eg, 'How often are you and your main partner in agreement about whether } \\
\text { to use condoms when you have sex with each other' rated on scale } 1=\text { never to } 5=\text { always). }\end{array}$ \\
\hline Communication $^{38} 39$ & $\begin{array}{l}\text { Self-report } 16 \text {-item assessment of participants' perceptions of how they deal with arguments and } \\
\text { communicate disagreements with their partner (eg, 'Exploding and getting out of control' rated on scale } \\
1=\text { very rarely to } 5=\text { very often). }\end{array}$ \\
\hline Collaborative coping ${ }^{40}$ & $\begin{array}{l}\text { Self-report 8-item assessment of participants' perception of how they and their partner cope with stress } \\
\text { (eg, 'I show my partner through my behavior when I am not doing well or when I have problems (1=very } \\
\text { rarely to } 5=\text { very often). }\end{array}$ \\
\hline
\end{tabular}

ART, antiretroviral therapy; CR-HIV, Composite Risk for HIV; DBS, dried blood spot; PrEP, pre-exposure prophylaxis. 
up and are asked questions to determine whether the intervention had an impact on their break up.

A computerised scheduling system tracks due dates and completion status of follow-ups. Participants receive a reminder about follow-up assessments seven and 2 days prior to the appointment. Each follow-up survey assesses relationship dissolution and measures of programme satisfaction. For those reporting relationship dissolution, questions assess whether the dissolution was linked to study participation or some other factor. Calls are made each day to participants who have not been scheduled or missed an appointment.

\section{Retention}

Multiple modes of contact are used to encourage follow-ups over 12 months. The team holds regular meetings to ensure study protocols and to examine retention rates. Should recruitment or retention rates deviate from anticipated rates, the team will brainstorm novel strategies and/or discuss strategies with the community advisory board. To reduce attrition, participants are paid for each visit. Additionally, participants receive business-sized cards with their next scheduled visit when at the research site and other messages on social media. In our prior trials we developed procedures to maintain at minimum $80 \%-90 \%$ follow-up rates. ${ }^{20} 50$

\section{Data management}

All data are password protected and backed up daily to an encrypted secure server. Electronic copies of the data are on a secure server and paper copies will be destroyed at the end of the study. Information provided by participants is coded with a number to help protect privacy; the link listing names with numbers is kept in locked files. Only study staff have access to study files. Reports and publications about the study will never refer to participants by name. All staff members are trained in procedures for maintaining confidentiality of participant information.

\section{Statistical methods}

\section{Outcomes}

Frequency tables for all variables and measures of central tendency and variability for continuous variables will characterise the sample and will be stratified by study arm to check for imbalances. If the intervention and control groups differ significantly at baseline on one or more covariates, we will use methods based on the Rubin causal model (eg, propensity scores, double-robust estimation) to obtain the desired marginal effect estimates under the counterfactual assumption of balanced groups. ${ }^{51-55}$

To address our primary aim, we hypothesise that the odds of CR-HIV will be lower for It Takes Two participants than for control participants. We will use general estimating equations (GEE) for the primary analysis to test our primary hypothesis by a planned time-averaged comparison of post-baseline measurements of CR-HIV from the intervention group with the post-baseline measures of CR-HIV from the control group. Alpha $(\alpha)$ will be set at 0.05 for this planned comparison. Any additional post-hoc comparisons (eg, paired comparisons of groups at each time point) will maintain nominal alpha of 0.05 through the use of simulation-based stepdown multiple comparison methods. ${ }^{56}$ Because each partner within a dyad may have a different CR-HIV value based on sexual behaviour with a new main partner or outside partners, repeated observations from individuals within dyads will be the unit of analysis. The alternating logistic regression approach implemented in SAS PROC GENMOD will be used to address the 3-level clustering of observations within participants and participants within dyads by treating dyads as clusters and participants as subclusters. Couple serostatus will be included as a covariate as required by the stratified randomised design.$^{57}$ Additional relevant covariates such as relationship length, relationship dissolution, as well as intersectionality criteria and structural drivers (eg, age, race/ethnicity, poverty, unemployment) will be pre-specified based on the literature and theory. This primary analysis will follow an intentionto-treat approach.

To explore the effect of the intervention on hypothesised mediators, analyses will explore whether participants assigned to It Takes Two report higher mean scores on theory-based constructs such as communication and mutual-solving strategies following the intervention. These analyses will also investigate whether these constructs at 3, 6 and 9 months mediate the relationship between intervention group assignment and CR-HIV at 6, 9 and 12 months, respectively, and whether couple HIV-serostatus and (in line with National Institutes of Health (NIH) guidelines to consider sex as a biological variable) whether gender moderate these associations. Mediation and moderation will be assessed using the causal inference-based approach of Valeri and VanderWeele, which yields optimal estimates of indirect effects in the presence of moderators and non-continuous outcomes and mediators.$^{58}$ Mplus will be used to fit causal mediation-moderation models because it can adjust standard errors for clustering of participants within couples. ${ }^{59}$

\section{Additional analyses}

It Takes Two is designed to be equally efficacious across intersectional subgroups of participants, so we do not anticipate differential intervention effects by subgroup. However, we will conduct additional exploratory sensitivity analyses to evaluate the moderating effects of age, HIV status, race/ethnicity, poverty and unemployment to explore whether these sociodemographic characteristics enhance or dampen the intervention's effects on CR-HIV and the theory-based mediators listed above. Where cell sizes permit, subgroup analyses will be performed, stratifying on race/ethnicity, age and poverty. While our study is not formally powered to examine subgroup differences and it would be infeasible to recruit sufficiently large numbers of participants in each category to test for differences formally, these analyses may illuminate trends in group differences, which can inform future studies of 
specific subpopulations if trends are detected. Moderation and subgroup analyses will be performed using the same GEE and causal inference approaches described earlier. $^{58}$

\section{Analysis population and missing data}

We will address incomplete data with multiple imputation (MI) ${ }^{60} 61$ because MI makes the relatively mild assumption that incomplete data arise from a conditionally missingat-random (MAR) mechanism. Auxiliary variables will be included to help meet the MAR assumption ${ }^{62}$; sensitivity analyses will be conducted with weighted MI to assess the MAR assumption's robustness ${ }^{63}$ using SAS version 9.4 and Mplus.

\section{Patient and public involvement}

The study design, procedures and measures were developed based on prior community-engaged research using qualitative focus group discussions and surveys with members of the target population, which revealed the need for couples-focused approaches to HIV prevention with trans women and their partners. ${ }^{7-9}$ A pilot study involving members of the community provided preliminary evidence and feedback regarding intervention content and approach. ${ }^{20} \mathrm{~A}$ community advisory board comprising community members and stakeholders is involved with informing design choices, recruitment and retention strategies, intervention content and dissemination.

\section{Monitoring \\ Data monitoring \\ Formal committee}

Our Data Safety Monitoring Board is independent from the sponsor; members do not have any competing interests. Board members were chosen for their relevant expertise on study content, population, and methodologies. The roles of our Data Safety Monitoring Board include reviewing analyses of data safety to determine whether the trial should continue as originally designed, should be changed or should be terminated based on these data; reviewing trial performance information, such as accrual information; determining whether and to whom results should be released prior to the reporting of the study results; reviewing reports of related studies to determine whether the monitored study needs to be changed or terminated; reviewing major proposed modifications to the study prior to their implementation; and providing study leadership with written summary information following board meetings. The chair of the board serves on a rotating basis in alphabetical order by name. There is no separate coordinating centre or steering committee.

\section{Interim analysis}

No interim analyses are planned.

\section{Harms}

Adverse events will be tracked and follow-through will be conducted via referrals and follow-up. An adverse event form has been developed to provide details of the incident, actions taken, supervisor notes and follow-up steps. The form, supplemented by regular study notes, will be sent immediately to appropriate agencies, including the Data Safety Monitoring Board chair, institutional review board (IRB) and NIH. Any action recommended by the Data Safety Monitoring Board or IRB will be conveyed to NIH.

\section{Auditing}

No trial audits are planned, but participating institutions have the authority to perform random audits of research protocols.

\section{ETHICS AND DISSEMINATION \\ Research ethics approval}

The University of California, San Francisco is the IRB of record. This study has been reviewed and approved by the Institutional Review Boards at the University of California, San Francisco (19-28624) and approved by University of Michigan (HUM00147690).

\section{Protocol amendments}

All proposed protocol modifications will be reviewed and approved by NIH prior to implementation of amendments. All modifications approved by NIH will be submitted to the University of California, San Francisco and University of Michigan IRB and the contact principal investigator will make the appropriate changes to the protocol via clinicaltrials.gov. If any major study changes are made, we will re-consent participants, as needed.

\section{Consent or assent}

Two consent processes take place; verbal informed consent is conducted for the eligibility visit, and signed informed consent is conducted at the baseline enrolment visit (see online supplemental file). Verbal informed consent for the eligibility visit takes place after both partners have been screened and express interest in participating in the study as a couple. If eligible and interested in participating as a couple, the participants are scheduled for the baseline enrolment visit. Assessors complete the consent procedures.

Signed informed consent for enrolment takes place at the baseline enrolment visit. If both members of the couple wish to consent, they each sign the informed consent form and are enrolled together as a couple.

\section{Confidentiality}

The following confidentiality protection steps are being implemented: study staff participate in training, and ongoing monitoring and supervision to ensure understanding of the ethical issues involved in this research; only trained staff will know the name, identification number and contact information of participants; consent forms are kept in locked files; personal identifiers linked to data are removed and replaced by code numbers in all records. Electronic copies of data are stored on a 
secure password-protected server. Paper copies of data will be destroyed at the end of the study. Participants are informed that their assessment data will not be shared with their partner.

\section{Declaration of interests}

There are no competing interests among the principal investigators.

\section{Access to data}

At the completion of the study, the full de-identified dataset and codebook will be shared via download link located at the University of Michigan Digital Repository. User registration and agreement to conditions of use will be required in order to access or download files.

\section{Ancillary and post-trial care}

During study enrolment, participants receive referrals to emergency health and psychological services if needed, including referrals to HIV confirmatory testing and care for those who receive an HIV-positive diagnosis. Participants receive a brochure with information about culturally appropriate and transgender-sensitive health, psychological and social services at enrolment. No provisions for post-trial care are planned.

\section{Dissemination policy}

\section{Trial results}

Following study completion and publication of primary reports, research data will be shared in accordance with NIH guidelines (http://grants.nih.gov/grants/policy/ data_sharing/).

As contact Principal Investigator, Dr Gamarel will share information about this trial via timely registration, updates and results reporting in ClinicalTrials.gov in accordance with NIH policy. The informed consent documents used for this trial includes statements to inform participants that information about the trial will be posted in clinicaltrials.gov.

\section{Authorship}

The Multiple Principal Investigators will follow International Committee of Medical Journal Editors guidelines for determining authorship eligibility and order. Final authorship decisions will be made by consensus among the principal investigators. No professional writers will be used.

\section{Reproducible research}

The full de-identified dataset and codebook will be shared at the University of Michigan Digital Repository.

\section{DISCUSSION}

To our knowledge, this is the first trial to test the efficacy of a couples-focused HIV prevention programme developed for trans women and their partners. The intervention is designed to facilitate empowerment in couples via knowledge and skills to choose from a range of empirically supported HIV prevention goals best suited to their relationship rather than focus exclusively on one particular HIV prevention option. The results of this study will provide insight as to how a theory-based dyadic approach can foster couple-level motivation for behaviour change and impact mechanisms of change including communication and joint problem-solving skills.

We updated and refined the original CHIP protocol to reflect the current HIV prevention best practice evidence by incorporating biomedical HIV prevention strategies. It Takes Two consists of four sessions, rather than three and includes new content on biomedical prevention and emphasises partner communication. We also expanded partner eligibility requirements to include trans women's partners of all genders to better reflect current partnerships in the USA. The primary outcome measure is CR-HIV, a novel composite variable which acknowledges the diversity of HIV prevention needs that couples might have and desire.

If the It Takes Two intervention demonstrates efficacy in comparison to an HIV prevention information control condition, there will be methodologically rigorous support for implementing this approach within HIV prevention and care settings in order to reduce disparities in HIV transmission and acquisition among some of the highest priority HIV prevention populations in the USA.

\section{Author affiliations}

${ }^{1}$ Department of Health Behavior and Health Education, University of Michigan School of Public Health, Ann Arbor, Michigan, USA

${ }^{2}$ Center for AIDS Prevention Studies, University of California, San Francisco, California, USA

${ }^{3}$ Division of Prevention Science, University of California, San Francisco, California, USA

${ }^{4}$ Department of Obstetrics, Gynecology, \& Reproductive Sciences, University of California, San Francisco, California, USA

${ }^{5}$ Public Health Institute, Oakland, California, USA

${ }^{6}$ Department of Health Behavior and Biological Sciences, University of Michigan School of Nursing, Ann Arbor, Michigan, USA

${ }^{7}$ Department of Behavioral and Social Sciences, Brown University School of Public Health, Providence, Rhode Island, USA

Acknowledgements We thank study staff members Danielle Castro, Deepalika Chakravarty, Carla Clynes, Samantha Dilworth, Willi Farrales, Akira Jackson, Wesley King, Breonna McCree, Azze Ngo, David Olem, Lance Pollack, Ellen Stein, Sophia Zamudio-Haas, and the UCSF Center of Excellence for Transgender Health Community Advisory Board for their involvement in informing design choices, recruitment and retention strategies, intervention content, and dissemination. We also grateful to Drs Susannah Allison and Gregory Greenwood for their support of this work.

Contributors KG, JS and D0 are multiple principal investigators and conceived the trial, initiated the study design and are grant holders. KG, JS, DO, TBN, TN, MOJ and LAD designed the study. TBN provided statistical expertise in clinical trial design and will perform statistical analyses. RLK oversaw formative qualitative data collection and compiled a preliminary draft of the study protocol manuscript. All authors contributed to the manuscript and consented to final publication.

Funding This work was supported by the National Institutes of Health grant number R01MH115765. KG was supported in part by the National Institutes of Health grant number R25MH067127.

Disclaimer Study design and content are the sole responsibility of the authors and do necessarily represent the official views of the sponsors.

Competing interests None declared. 
Patient and public involvement Patients and/or the public were involved in the design, or conduct, or reporting, or dissemination plans of this research. Refer to the Methods section for further details.

Patient consent for publication Not required.

Provenance and peer review Not commissioned; externally peer reviewed.

Open access This is an open access article distributed in accordance with the Creative Commons Attribution Non Commercial (CC BY-NC 4.0) license, which permits others to distribute, remix, adapt, build upon this work non-commercially, and license their derivative works on different terms, provided the original work is properly cited, appropriate credit is given, any changes made indicated, and the use is non-commercial. See: http://creativecommons.org/licenses/by-nc/4.0/.

ORCID iD

Don Operario http://orcid.org/0000-0003-2467-5048

\section{REFERENCES}

1 Poteat T, German D, Kerrigan D. Managing uncertainty: a grounded theory of stigma in transgender health care encounters. Soc Sci Med 2013;84:22-9.

2 Baral SD, Poteat T, Strömdahl S, et al. Worldwide burden of HIV in transgender women: a systematic review and meta-analysis. Lancet Infect Dis 2013;13:214-22.

3 Becasen JS, Denard CL, Mullins MM, et al. Estimating the prevalence of HIV and sexual behaviors among the US transgender population: a systematic review and meta-analysis, 2006-2017. Am J Public Health 2019;109:e1-8.

4 Herbst JH, Jacobs ED, Finlayson TJ, et al. Estimating HIV prevalence and risk behaviors of transgender persons in the United States: a systematic review. AIDS Behav 2008;12:1-17.

5 Clark H, Babu AS, Wiewel EW, et al. Diagnosed HIV infection in transgender adults and adolescents: results from the National HIV surveillance system, 2009-2014. AIDS Behav 2017;21:2774-83.

6 Melendez RM, Pinto R. 'It's really a hard life': love, gender and HIV risk among male-to-female transgender persons. Cult Health Sex 2007;9:233-45.

7 Nemoto T, Operario D, Keatley J, et al. Social context of HIV risk behaviours among male-to-female transgenders of colour. AIDS Care 2004;16:724-35.

8 Nemoto T, Operario D, Keatley J, et al. HIV risk behaviors among male-to-female transgender persons of color in San Francisco. Am J Public Health 2004:94:1193-9.

9 Operario D, Nemoto T, Iwamoto M, et al. Unprotected sexual behavior and HIV risk in the context of primary partnerships for transgender women. AIDS Behav 2011;15:674-82.

10 Reisner SL, Mimiaga MJ, Bland S, et al. Hiv risk and social networks among male-to-female transgender sex workers in Boston, Massachusetts. J Assoc Nurses AIDS Care 2009;20:373-86.

11 Reisner SL, Menino D, Leung K, et al. "Unspoken Agreements": Perceived Acceptability of Couples HIV Testing and Counseling (CHTC) Among Cisgender Men with Transgender Women Partners. AIDS Behav 2019;23:366-74.

12 El-Bassel N, Gilbert L, Witte S, et al. Couple-based HIV prevention in the United States: advantages, gaps, and future directions. J Acquir Immune Defic Syndr 2010;55 Suppl 2:S98-101.

13 Wu E, El-Bassel N, McVinney LD, et al. Feasibility and promise of a couple-based HIV/STI preventive intervention for methamphetamineusing, black men who have sex with men. AIDS Behav

14 El-Bassel N, Witte SS, Gilbert L, et al. The efficacy of a relationshipbased HIV/STD prevention program for heterosexual couples. Am J Public Health 2003;93:963-9.

15 Crepaz N, Tungol-Ashmon MV, Vosburgh HW, et al. Are couplebased interventions more effective than interventions delivered to individuals in promoting HIV protective behaviors? A meta-analysis. AIDS Care 2015;27:1361-6.

16 Reisner SL. Project LUST: adapting couples HIV counseling and testing $(\mathrm{CHCT})$ for transgender women and their cisgender male sexual partners, in Harvard University CFAR conference presentation. Boston, MA, 2015.

17 Operario D, Nemoto T, Iwamoto M, et al. Risk for HIV and unprotected sexual behavior in male primary partners of transgender women. Arch Sex Behav 2011;40:1255-61.

18 Gamarel KE, Reisner SL, Laurenceau J-P, et al. Gender minority stress, mental health, and relationship quality: a dyadic investigation of transgender women and their cisgender male partners. J Fam Psychol 2014;28:437-47.

19 Gamarel KE, Reisner SL, Darbes LA, et al. Dyadic dynamics of HIV risk among transgender women and their primary male sexual partners: the role of sexual agreement types and motivations. AIDS Care 2016;28:104-11.

20 Operario D, Gamarel KE, Iwamoto M, et al. Couples-focused prevention program to reduce HIV risk among transgender women and their primary male partners: feasibility and promise of the couples HIV intervention program. AIDS Behav 2016;21.

21 Reisner SL, Gamarel KE, Nemoto T, et al. Dyadic effects of gender minority stressors in substance use behaviors among transgender women and their non-transgender male partners. Psychol Sex Orientat Gend Divers 2014;1:63-71.

22 Mitchell JW, Wu Y, Gamarel KE. Timing and sequence of events: HIV status disclosure, condomless anal sex, and sexual agreement formation among same-sex male couples. J Sex Res 2019;56:728-39.

23 Parsons JT, Starks TJ, Gamarel KE, et al. Non-monogamy and sexual relationship quality among same-sex male couples. J Fam Psychol 2012;26:669-77.

24 Gass K, Hoff CC, Stephenson R, et al. Sexual agreements in the partnerships of internet-using men who have sex with men. AIDS Care 2012;24:1255-63.

25 Hoff CC, Beougher SC, Chakravarty D, et al. Relationship characteristics and motivations behind agreements among gay male couples: differences by agreement type and couple serostatus. AIDS Care 2010;22:827-35.

26 Grant RM, Lama JR, Anderson PL, et al. Preexposure chemoprophylaxis for HIV prevention in men who have sex with men. N Engl J Med 2010;363:2587-99.

27 Cohen MS, Chen YQ, McCauley M, et al. Prevention of HIV1 infection with early antiretroviral therapy. $N$ Engl J Med 2011;365:493-505.

28 Cohen MS, McCauley M, Gamble TR. HIV treatment as prevention and HPTN 052. Curr Opin HIV AIDS 2012;7:99-105

29 Gamarel KE, Chakravarty D, Neilands TB, et al. Composite risk for HIV: a new approach towards integrating biomedical and behavioral strategies in Couples-Based HIV prevention research. AIDS Behav 2019;23:283-8.

30 Chan A-W, Tetzlaff JM, Altman DG, et al. Spirit 2013 statement: defining standard protocol items for clinical trials. Ann Intern Med 2013;158:200-7.

31 CDC. HIV surveillance report, diagnoses of HIV infection in the United States and dependent areas, 2015.

32 California Department of Public Health. The California HIV surveillance report. Sacramento, CA: Center for Infectious Diseases, Office of AIDS, 2017.

33 San Francisco AIDS Foundation. Transgender persons and HIV. San Francisco, CA, 2009.

34 McMahon JM, Tortu S, Torres L, et al. Recruitment of heterosexual couples in public health research: a study protocol. BMC Med Res Methodol 2003;3:24.

35 Syvertsen JL, Robertson AM, Abramovitz D, et al. Study protocol for the recruitment of female sex workers and their non-commercial partners into couple-based HIV research. BMC Public Health 2012;12:136.

36 Castillo-Mancilla J. Emtricitabine-triphosphate in dried blood spots as a marker of recent dosing. antimicrobial agents and chemotherapy, 2016.

37 Gandhi M, Glidden DV, Liu A, et al. Strong correlation between concentrations of tenofovir (TFV) emtricitabine (FTC) in hair and TFV diphosphate and FTC triphosphate in dried blood spots in the iPrEx open label extension: implications for pre-exposure prophylaxis adherence monitoring. J Infect Dis 2015;212:1402-6.

38 Kurdek LA. Conflict resolution styles in gay, lesbian, heterosexua nonparent, and heterosexual parent couples. J Marriage Fam 1994;56:705-22.

39 Bonache H, Ramírez-Santana G, Gonzalez-Mendez R. Conflict resolution styles and teen dating violence. Int $J$ Clin Health Psychol 2016;16:276-86.

40 Bodenmann G. Dyadic coping inventory (DCl). test manual. Bern Göttingen: Huber Testverlag, 2008.

41 Gamarel KE, Golub SA. Sexual goals and perceptions of goal congruence in individuals' PrEP adoption decisions: A mixedmethods study of gay and bisexual men who are in primary relationships. Ann Behav Med 2020;54:237-48.

42 NCSS. NCSS PASS[computer program. Version 2008. Kaysville,Utah: NCSS Statistical Software, 2008.

43 Diggle PJ, Liang KY, Zeger SL. Analysis of longitudinal data. New York: Oxford University, 1994.

44 Sevelius JM, Patouhas E, Keatley JG, et al. Barriers and facilitators to engagement and retention in care among transgender women living with human immunodeficiency virus. Ann Behav Med 2014:47:5-16. 
45 Gamarel KE, Comfort M, Wood T, et al. A qualitative analysis of male couples' coping with HIV: Disentangling the "we". J Health Psychol 2016;21:2125-37.

46 Saberi P, Gamarel KE, Neilands TB, et al. Ambiguity, ambivalence, and apprehensions of taking HIV-1 pre-exposure prophylaxis among male couples in San Francisco: a mixed methods study. PLoS One 2012;7:e50061.

47 Conroy AA, Gamarel KE, Neilands TB, et al. Relationship dynamics and partner beliefs about viral suppression: a longitudinal study of male couples living with HIV/AIDS (the Duo project). AIDS Behav 2016;20:1572-83.

48 Gamarel KE, Neilands TB, Conroy AA, et al. A longitudinal study of persistent smoking among HIV-positive gay and bisexual men in primary relationships. Addict Behav 2017;66:118-24.

49 Gamarel KE, Starks TJ, Dilworth SE, et al. Personal or relational? examining sexual health in the context of HIV serodiscordant samesex male couples. AIDS Behav 2014;18:171-9.

50 Lelutiu-Weinberger C, Pachankis JE, Gamarel KE, et al. Feasibility, acceptability, and preliminary efficacy of a Live-Chat social media intervention to reduce HIV risk among young men who have sex with men. AIDS Behav 2015;19:1214-27.

51 Luellen JK, Shadish WR, Clark MH. Propensity scores: an introduction and experimental test. Eval Rev 2005;29:530-58.

52 Rubin DB. On principles for modeling propensity scores in medical research. Pharmacoepidemiol Drug Saf 2004;13:855-7.

53 Seaman S, Copas A. Doubly robust generalized estimating equations for longitudinal data. Stat Med 2009;28:937-55.

54 Shadish WR, Luellen JK, Clark M. Propensity scores and QuasiExperiments: a testimony to the practical side of Lee Sechrest, 2006.

55 Weitzen S, Lapane KL, Toledano AY, et al. Principles for modeling propensity scores in medical research: a systematic literature review. Pharmacoepidemiol Drug Saf 2004;13:841-53.

56 Westfall P, Young S. Resampling-Based multiple testing: examples and methods for $p$-Value adjustment. New York: John Wiley and Sons, 1993.

57 Kahan BC, Morris TP. Improper analysis of trials randomised using stratified blocks or minimisation. Stat Med 2012;31:328-40.

58 Valeri L, VanderWeele TJ. Mediation analysis allowing for exposuremediator interactions and causal interpretation: theoretical assumptions and implementation with SAS and SPSS macros. Psychol Methods 2013;18:137-50.

59 Muthén B. Applications of causally Dened direct and indirect effects in mediation analysis using SEM in mPLUS, 2011. Available: http:// www.statmodel.com/examples/penn.shtml

60 Little RJ, Rubin DB. Statistical analyses with missing data. 3rd ed. Hoboken, New Jersey: John Wily \& Sons, 2020.

61 Meolenberghs G, Fitzmaurice G, Kenward MG, et al. Handbook of missing data methodology. Boca Raton, Florida: Taylor \& Francis Group, 2015.

62 Collins LM, Schafer JL, Kam CM. A comparison of inclusive and restrictive strategies in modern missing data procedures. Psychol Methods 2001;6:330-51.

63 Carpenter JR, Kenward MG, White IR. Sensitivity analysis after multiple imputation under missing at random: a weighting approach. Stat Methods Med Res 2007;16:259-75.

64 Reback CJ, Fletcher JB. HIV prevalence, substance use, and sexual risk behaviors among transgender women recruited through outreach. AIDS Behav 2014;18:1359-67.

65 Wilson IB, Fowler FJ, Cosenza CA, et al. Cognitive and field testing of a new set of medication adherence self-report items for HIV care. AIDS Behav 2014;18:2349-58.

66 Hoff CC, Chakravarty D, Bircher AE, et al. Attitudes towards PrEP and anticipated condom use among concordant HIV-negative and HIV-discordant male couples. AIDS Patient Care STDS 2015;29:408-17.

67 Stall R, Hoff C, Coates TJ, et al. Decisions to get HIV tested and to accept antiretroviral therapies among gay/bisexual men: implications for secondary prevention efforts. J Acquir Immune Defic Syndr Hum Retrovirol 1996;11:151-60.

68 Bohns VK, Lucas GM, Molden DC, et al. Opposites fit: regulatory focus complementarity and relationship well-being. Soc Cogn 2013;31:1-14.

69 Gamarel KE, Golub SA. Sexual goals and perceptions of goal congruence in individuals' PrEP adoption decisions: A mixedmethods study of gay and bisexual men who are in primary relationships. Ann Behav Med 2020;54:237-48. 